\section{PO 8495 DATA SHARING IS PART OF DATA MANAGEMENT: THE NEED FOR A HOLISTIC AND COHERENT VIEW ON RESEARCH DATA MANAGEMENT}

${ }^{1}$ Harry Van Loen*, ${ }^{2}$ Mary Thiongo, ${ }^{1} Y$ ven Van Herrewege. ${ }^{1}$ Institute of Tropical Medicine Antwerp, Belgium; ${ }^{2}$ International Centre for Reproductive Health Kenya, Mombasa, Kenya

\subsection{6/bmjgh-2019-EDC.120}

Background Awareness of data management (DM) is often restricted to 'the cost of computers' or 'the need for a database'. Recently, 'data sharing' can be added to this shortlist. Indeed, in recent years data sharing became often required or so strongly promoted that the importance of all other aspects related to DM or data handling in clinical tended still to be overlooked. However, the development of data sharing guidelines and associated privacy regulations (e.g. the EU General Data Protection Regulation) created a new momentum for highlighting the importance of qualitative data management.

Methods An overview of DM processes is given, within the framework and challenges of conducting non-commercial clinical trials in North-South partnerships.

Results The DM workflow of a clinical trial is presented, highlighting essential DM tasks, deliverables and milestones. Pre-study tasks and deliverables are addressed: SOPs, a data management plan, the implementation of a GCP-compliant validated data management system and compliance to data quality, privacy, security and standards (e.g. MedDRA, CDISC). Subsequent study-specific processes including the collection, entry, querying and cleaning of the data are discussed. In addition, DM metrics important to guide quality, productivity and timelines are reviewed while considering their impact on post-study activities such as data sharing.

Conclusion Data sharing is only one of many DM tasks, at the end of the DM workflow. Focusing too much on data sharing while neglecting other DM aspects might lead to underestimating the workload, resources, quality assurance and time needed for data management and by and large for the trial itself. Integrating data sharing into a holistic vision on data management is paramount for clinical research.

\section{PO 8496 ACCURACY OF DIAGNOSIS AND HAEMATOLOGICAL DIFFERENCE AMONG MALARIA PATIENTS IN RURAL AND URBAN AREAS IN THE ASHANTI REGION OF GHANA}

Abdul-Hakim Mutala, Kingsley Badu, Austine Tweneboah, Samul Agordzo, Dawood Ackom Abbas. Department of Theoretical and Applied Biology, Kwame Nkrumah University of Science and Technology (KNUST), Kumasi, Ghana

\subsection{6/bmjgh-2019-EDC.121}

Background Over recent years, there has been an increase in the use of a histidine-rich protein 2(HRP-2)-based rapid diagnostic test (RDT) in the diagnosis of malaria. Accurate and prompt diagnosis of malaria will help reduce parasite reservoir and reduce malaria transmission. However, the underdiagnosis of malaria due to low parasite density hinders malaria eradication. The study aimed at establishing the baseline information on the accuracy of the HRP2-based RDT used in Ghana in three communities (Agona [rural], Kuntanase [peri-urban] and Kumasi [urban]) while determining the haematological difference among malaria patients.

Methods Cross-sectional study was conducted from January to April 2018. A total of 304 participants were recruited in the study. Microscopy and RDT were used in the detection of malaria parasitaemia in all the samples.

Results The overall sensitivity, specificity, negative predictive value and positive predictive value was 75.9\%, 95.6\%, 64.7\% and $97.4 \%$ respectively. The HRP-2 based RDT was highly sensitive $(100 \%)$ for parasite density $\geq 250$ parasite $\mu$ l and relatively low for parasite density $\leq 100$ parasite/ $\mu$ l $(50 \%$ Kumasi, 67\%- Agona and 75\%- Kuntanase). On the other hand, Agona (rural) recorded the highest prevalence (15.8\%) followed by Kumasi (urban) (9\%) and Kuntanase (peri-urban) being the lowest $(6.8 \%)$. The difference in prevalence was however not statistically significant across the three communities. The rural area also accounted for highest parasite density (mean 99.53) and lowest in urban (60.29) with a statistical difference $(\mathrm{p}<0.001)$. The difference in white blood cell levels was significant $(<0.0001)$ across Agona, Kuntanase and Kumasi. RBC and $\mathrm{Hb}$ levels were however not significant.

Conclusion The high specificity observed indicates that the majority of the patients without malaria were correctly diagnosed. Notwithstanding, the sensitivity was relatively low and below the WHO standard of $\geq 95 \%$ hence a significant number of malaria-positive cases were misdiagnosed. It is therefore important that the accuracy of RDT should be frequently assessed to improve its quality.

\section{PO 8502 DETERMINANTS AND PREVALENCE OF PARASITE RESISTANCE AMONG PREGNANT WOMEN RECEIVING IPTP WITH SULPHADOXINE-PYRIMETHAMINE IN NIGERIA}

${ }^{1}$ Atinuke Olaleye, ${ }^{2}$ Noblefather Uyaiabasi, ${ }^{3}$ Charles Elikwu, ${ }^{2}$ Oladapo Walker. ${ }^{1}$ Dept of Obstetrics and Gynecology, Babcock University, Ilishan-Remo, Ogun State, Nigeria; ${ }^{2}$ Dept of Pharmacology, Babcock University, llishan-Remo, Ogun State, Nigeria; ${ }^{3}$ Dept of Medical Microbiology, Babcock University, llishan-Remo, Ogun State, Nigeria

\subsection{6/bmjgh-2019-EDC.122}

Background Malaria in pregnancy carries a risk of significant adverse maternal and infant outcomes. Intermittent preventive treatment in pregnancy (IPTp) is advocated to reduce its occurrence, but resistance to sulphadoxine-pyrimethamine (SP) is being reported. This study aims to describe the burden of SP resistance and determinants of its occurrence among pregnant women receiving IPTp in Nigeria.

Methods A prospective observational study is to be conducted in Ogun State over 24 months. Pregnant women 16-28 weeks gestation meeting the eligibility criteria are being enrolled; blood samples are taken for analysis pre- and post- IPTp-SP administration at scheduled intervals. Microscopy-confirmed parasitaemic samples will be analysed using PCR to detect drug resistance markers (pfdhfr and pfdhps). Participants will be followed up until 28 days post-delivery and assessed for maternal and foetal outcomes (anaemia, low birth weight, preterm delivery, placental parasitaemia, stillbirth, neonatal death). The primary endpoint is the prevalence of the SP resistance gene markers. Secondary endpoints include the prevalence of peripheral and placental parasitaemia at delivery; incidence of maternal and newborn morbidity; parasitaemia pre-IPTp and day 28 post-IPTp; risk factors for SP resistance and haemoglobin changes at delivery.

Results Following statistical analysis with STATA 14, results will be displayed in appropriate formats. Geometric mean parasite densities with 95\% confidence intervals will be calculated, and proportions compared using the t-test, Chi-square 